\title{
La prise de Saint-Pierre-et-Miquelon par les forces de la France libre : Noël 1941
}

\section{William Hanna}

Volume 16, numéro 3, décembre 1962

URI : https://id.erudit.org/iderudit/302212ar

DOI : https://doi.org/10.7202/302212ar

Aller au sommaire du numéro

Éditeur(s)

Institut d'histoire de l'Amérique française

ISSN

0035-2357 (imprimé)

1492-1383 (numérique)

Découvrir la revue

Citer cet article

Hanna, W. (1962). La prise de Saint-Pierre-et-Miquelon par les forces de la France libre : Noël 1941. Revue d'histoire de l'Amérique française, 16(3), 369-387. https://doi.org/10.7202/302212ar d'utilisation que vous pouvez consulter en ligne.

https://apropos.erudit.org/fr/usagers/politique-dutilisation/ 


\section{LA PRISE DE SAINT-PIERRE-ET-MIQUELON PAR LES FORCES DE LA FRANCE LIBRE :}

\section{NOËL 1941}

Le 24 décembre 1941, un détachement des forces de la «France libre», sous le commandement du vice-amiral Émile Henri Muselier, prit possession des îles Saint-Pierre-et-Miquelon alors sous le contrôle du gouvernement de Vichy. La presse et la radio mondiale diffusèrent la nouvelle (cette dernière fut relatée en première page du New York Times) et immédiatement les rapports se tendirent entre les puissances intéressées (selon Pickersgill, cela «créa une antipathie persistante entre Cordell Hull et Churchill »). ${ }^{1}$ Dans un documentaire présenté en 1961 par les émissions télévisées de la Canadian Broadcasting Corporation, l'événement fut cité comme « le plus beau cadeau de Noël que le monde ait eu cette année-là $\gg^{2}$ La prise de possession des îles et les contacts diplomatiques qui l'ont précédée et suivie sont cependant peu connus du public.

Plusieurs sources originales et secondaires mentionnent brièvement l'incident Saint-Pierre-et-Miquelon; certaines l'exploitent sur la base d'arguments spécieux tandis que d'autres n'en rapportent qu'une vue incomplète. Le présent article analyse le cadre politique et géographique de l'action, les événements et attitudes qui l'ont précédée, la prise de l'archipel et les réactions mondiales qui en ont découlé.

1 J. W. Pickersgill, The Mackenzie King Record (2 vol., Toronto, 1960), I: 318.

2 Close-Up, 17 décembre 1961. 


\section{CADRE DE L'ACTION}

Le début de la seconde guerre mondiale fut marqué par l'invasion de la Pologne en septembre 1939 par les armées allemandes. Les Pays-Bas, la Belgique et le Luxembourg devaient être les victimes suivantes des Nazis et le 22 juin 1940, le gouvernement de Vichy devait signer l'armistice. L'archipel Saint-Pierre-et-Miquelon acquit son importance dans le cadre de l'effort de guerre allié.

\section{Les Iles}

Le groupe d'îles Saint-Pierre-et-Miquelon, la plus ancienne possession française d'outremer, est situé au large du golfe du Saint-Laurent, à $32 \mathrm{~km}$. au sud des côtes de Terre-Neuve. Les habitants de l'archipel, dont la superficie couvre $240 \mathrm{~km} .^{2}$, sont presque exclusivement d'ascendance française. Les Français débarquèrent sur les îles au $16^{\text {ème }}$ siècle et après de courtes périodes de domination britannique, n'ont cessé d'occuper l'archipel depuis 1814.

La pêche constitue à présent l'industrie principale des îles dont la population compte au total 4,600 habitants, mais il n'en a pas toujours été ainsi. Au cours de la période de prohibition aux Etats-Unis, des profits considérables furent réalisés par des intermédiaires du commerce de liqueurs entre l'Europe et les Etats-Unis; cependant ce trafic fut interdit et l'industrie de la pêche reprit son essor.

\section{Les Iles et la Guerre}

Les habitants de l'archipel ont une réputation de fervents patriotes. La nouvelle du désastre français de juin 1940 fut reçue avec angoisse; une messe spéciale fut célébrée et toute activité commerciale cessa. ${ }^{3}$ Après l'armistice, Saint-Pierre-etMiquelon passait sous le régime du gouvernement Pétain et du Gouverneur-résident, le baron de Bournat, marié à une Allemande et probablement un puissant partisan du gouvernement de

${ }^{3}$ F. K. Arnold, «Islands Adrift: St. Pierre and Miquelon», (National Geographic Magazine, LXXX, (décembre, 1934), 743-768): 766. 
Vichy. ${ }^{4} \mathrm{Vu}$ le rôle dominant joué par l'Allemagne nazie dans le gouvernement de Vichy, l'archipel ne manqua pas d'être utilisé à l'appui de l'effort de guerre de l'Axe. Ceci se réalisa de plusieurs façons.

Tout d'abord, une grande partie des produits de pêche locaux devait être envoyée à travers le blocus allié vers la France et de là vers l'Allemagne, contribuant dans une large proportion au ravitaillement de celle-ci. Certains navires furent arrêtés par les Anglais et l'équipage fut mis à la disposition des forces alliées; ce ne fut pas le cas pour la majorité des bâtiments.

En second lieu, l'utilisation des moyens de communications de l'archipel devait se révéler de grande importance. SaintPierre constitue une des stations du câble transatlantique et est pourvue d'un puissant émetteur radio. Par le truchement de ce dernier, les sympathisants de Vichy étaient à même d'informer la flotte sous-marine allemande des conditions météorologiques de la progression des convois. En outre, la station radio était un centre de diffusion de la propagande de l'Axe et un moyen de liaison avec les agents secrets pour l'envoi et la réception de messages chiffrés dans l'hémisphère occidental.

Les îles enfin devaient être d'une importance politique non négligeable. En juin 1940, le général Charles de Gaulle avait formé à Londres l'organisation de la «France libre » et désirait la voir reconnue comme le gouvernement français légitime. Cependant le gouvernement des Etats-Unis et la plupart des gouvernements alliées reconnaissaient toujours le gouvernement de Vichy et n'avaient pas l'intention de se retourner vers de Gaulle, en raison des avantages à escompter de bonnes relations avec Vichy, et étant donné le peu de confiance et de soutien dont l'organisation de de Gaulle jouissait à ce moment.

La «France libre » désirait être reconnue parce qu'un gouvernement français complètement indépendant serait un symbole important de résistance pour le peuple français et que le général de Gaulle se sentirait dès lors plus libre dans la

${ }^{4}$ Dans Close-Up, il nia favoriser Vichy. 
poursuite de certains objectifs, notamment en Afrique du Nord. Une intervention à Saint-Pierre-et-Miquelon apparut donc comme un moyen de "remuer le fond des choses, comme on jette une pierre dans l'étang $\gg .^{5}$

\section{PLANS MILITAIRES ET ÉCHANGES DIPLOMATIQUES}

Des pourparlers au sujet de Saint-Pierre-et-Miquelon furent entamés indépendamment à Ottawa et à Londres vers la mi-1940. Tout d'abord, au début de juin 1940, l'ambassadeur américain Moffat discuta l'occupation des îles avec les autorités canadiennes; au cours de ces conversations, le ministre canadien de la défense aérienne, C. G. Power, déclara que «si cela dépendait de lui, les troupes canadiennes occuperaient l'archipel ». Ensuite, le 5 juillet 1940, Moffat fit appel au premier ministre Mackenzie King et exprima le vœu que le Canada n'entreprît pas une occupation unilatérale des îles, étant donnée la situation délicate avec Vichy. King admit qu'il avait été l'objet de pressions de la part de Terre-Neuve, mais il donna l'assurance que des troupes ne seraient pas envoyées. ${ }^{6}$ Comme les Américains, il craignait que toute intervention directe dans les îles ne mît en danger la situation diplomatique internationale, déjà trop délicate.

La situation à Saint-Pierre-et-Miquelon fut aussi l'objet de conversations à Londres parmi les partisans de la «France libre ». Dès le début de juillet 1940, le général de Gaulle et l'amiral Muselier avaient considéré le problème de la libération de Saint-Pierre-et-Miquelon. Mais ni la liberté politique ni les moyens militaires à pareille action n'étaient disponibles immédiatement, surtout après que le gouvernement de Vichy eût envoyé un aviso vers l'archipel. Auparavant, au moment de l'affaire Mersel-Keber, ils avaient envisagé l'occupation des îles et une résistance symbolique désespérée, mais la guerre

${ }^{5}$ Charles de Gaulle, Mémoires de guerre: l'Appel 19140-1942 (Paris, 1954) : 184.

6 J. P. Moffat, The Moffat Papers (Cambridge, Massachusetts, 1956), $315,322,358$, et passim. 
n'avait pas été déclarée entre la France et l'Angleterre; la décision devait être retardée. Néanmoins, des préparatifs furent entamés pour la libération pacifique de Saint-Pierre-etMiquelon. ${ }^{7}$

Les relations diplomatiques cordiales de jure et de facto entretenues par plusieurs gouvernements alliés avec le gouvernement de Vichy constituaient les obstacles principaux à toute action de la part de la «France libre ». L'inquiétude du gouvernement Pétain au sujet des colonies françaises dans l'hémisphère occidental ne manquait pas de se faire sentir dans les communications secrètes franco-américaines, ignorées des Français de la «France libre ». En partie pour faire écho à cette inquiétude, le président Roosevelt annonça à la fin juillet que les Etats-Unis ne reconnaîtraient pas de changement de souveraineté des colonies des puissances européennes dans l'hémisphère occidental, et que si un tel changement devait affecter une colonie, des mesures seraient prises pour placer celle-ci sous protectorat interaméricain pour la durée de la guerre. Vichy fut davantage rassuré quand Roosevelt affirma qu'il entendait voir les colonies françaises conserver leur neutralité.

Les conversations entre les Etats-Unis et Vichy sur les colonies dans l'hémisphère occidental se poursuivirent jusqu'en 1942, mais celles qui furent tenues au cours des derniers mois de 1940, sont spécialement importantes. Elles aident à comprendre la position des Etats-Unis lorsque plus tard la « France libre » dut entrer en contact avec eux au sujet d'une intervention dans l'archipel. Les États-Unis prirent l'initiative de rouvrir les conversations avec Vichy quand le département de la Marine annonça, le $1^{\text {er }}$ octobre 1940, que le gouvernement de Vichy avait

7 E. H. Muselier, De Gaulle contre le Gaullisme (Paris, 1946), 247248 et passim. Quelques éclaircissements sont nécessaires, si l'on veut connaître exactement les relations entre l'amiral Muselier et le général de Gaulle, principalement si l'on consulte l'un et l'autre dans leurs écrits; en septembre 1940, la situation au sein des partisans de la «France libre» à Londres était devenue chaotique. Muselier n'avait jamais été en bons termes avec de Gaulle et sans nul doute il essaya de lui dérober la direction du mouvement. La lutte entre ces deux hommes devint si âpre que les Britanniques crurent une intervention nécessaire, ce qu'ils firent le 24 septembre. On permit à de Gaulle de garder la direction du mouvement, mais soupçons et dissensions n'en persistèrent pas moins parmi les chefs. 
alloué des sommes importantes pour des constructions militaires en Martinique. Roosevelt fit immédiatement appeler l'ambassadeur de Vichy à Washington, Henry Haye, et l'avertit que le statu quo devait être préservé aux colonies; il proposa que Vichy déclare publiquement la neutralité de ses possessions dans l'hémisphère occidental. En échange, Roosevelt offrit de dégeler du compte français les fonds nécessaires pour couvrir les frais des missions diplomatiques françaises aux États-Unis. ${ }^{8}$

Le 7 octobre, Henry Haye assura Sumner Welles qu'aucune construction militaire n'avait été effectuée depuis l'armistice et que le gouvernement français était prêt à coopérer de toutes les manières possibles. Plus précisément, il informa Welles que: $1^{\circ}$ Vichy accepterait la présence d'observateurs américains dans les colonies et qu'ils seraient aidés dans leur mission. $2^{\circ} \mathrm{L}$ 'amiral Robert, haut-commissaire de Vichy aux Antilles et à la Guadeloupe, recevrait instruction d'inviter un officier américain à étudier les problèmes qui avaient soulevé des inquiétudes dans les milieux américains; $3^{\circ}$ les fonctions de l'amiral Robert seraient étendues aux îles Saint-Pierre-et-Miquelon; et $4^{\circ}$ les colonies seraient démilitarisées. ${ }^{9}$

L'amiral américain Greenslade discuta ces points avec l'amiral Robert et apparemment ils aboutirent à un modus vivendi. Le représentant américain accepta de ne pas exiger une déclaration publique de neutralisation et Robert promit de se conformer méticuleusement aux modalités de l'accord, bien qu'il tînt à éviter un traité écrit, vu les difficultés de la situation internationale. Cependant Vichy retira soudainement son offre d'inviter des observateurs, et l'accord final prévoya uniquement la garantie du maintien du statu quo.

Le 13 décembre, le président Roosevelt envoya le message suivant au maréchal Pétain: "Vous pouvez être assuré que dans les circonstances actuelles, le gouvernement des États-Unis res-

$8 \mathrm{~W}$. L. Langer et S. E. Gleason, The Undeclared War (New York, 1953), 72 et passim.

$9 \mathrm{~F}$. Charles-Roux, Cinq mois tragiques aux affaires étrangères (Paris, 1949), 171-178. Voir aussi Langer and Gleason, The Undeclared War, $72-73$. 
tera fidèle aux accords conclus par nos deux gouvernements et regardant le maintien du statu quo des possessions françaises dans l'hémisphère occidental.» ${ }^{10}$

À la fin août 1940, par suite du manque de liberté d'action de la «France libre», l'amiral Muselier attira l'attention de l'amirauté britannique sur le problème de Saint-Pierre-et-Miquelon. Ceci incita le premier ministre Winston Churchill à donner au général de Gaulle l'assurance que le Royaume-Uni assumerait la responsabilité de la défense navale de toutes les colonies françaises ralliées à de Gaulle, spécialement si elles venaient à être attaquées par Vichy. ${ }^{11}$ Assuré de l'appui britannique, le général de Gaulle déclara le 19 septembre qu'il était temps de se préparer à prendre possession des colonies françaises d'Amérique. ${ }^{12}$ Le 23 octobre cependant, l'amirauté britannique fit savoir à l'amiral Muselier que, pour des raisons politiques, il serait peu sage d'occuper immédiatement Saint-Pierre-et-Miquelon. ${ }^{13}$ Ainsi l'année 1940 prit-elle fin sans que l'archipel ne fut libéré malgré l'insistance répétée des partisans de la «France libre».

Les partisans de la «France libre » poursuivirent les préparations militaires en vue de l'opération et, vers la mi-1941, ces dernières étaient terminées. Cependant, les complications politiques n'étant point résolues, il fut décidé de se tenir prêt et de retarder toute action dans l'espoir d'une amélioration de la situation diplomatique. Entre-temps, Saint-Pierre-et-Miquelon avait attiré l'attention des capitales alliées, principalement d'Ottawa. Avec la progression des hostilités, les Canadiens s'étaient de plus en plus rendus compte du danger qu'offrait à l'effort de guerre allié, le système de communication de Saint-Pierre. Le 3 novembre 1941, Norman Robertson, sous-secrétaire d'État cana-

${ }^{10}$ W. L. Langer, Our Vichy Gamble (New York, 1947), 216-217. La conception du statu quo et de la souveraineté par la «France libre » fut exposée par le baron de Vaux, délégué au Comité pour la «France libre 》 au Caire, lorsqu'il écrivait qu'il n'y avait « aucun changement de souveraineté mais... au contraire, confirmation de la souveraineté française ». Cf., de Gaulle, Mémoires de guerre, I: 507-508. De Gaulle estimait qu'une intervention par la «France libre» dans une possession de Vichy constituait « une affaire intérieure française ». Ibid: 184.

11 Muselier, De Gaulle, 248-249.

12 Ibid., 249.

13 Ibid., 250. 
dien aux Affaires Etrangères, informa J. P. Moffat de l'arrivée prochaine et possible à Saint-Pierre de chargés de mission qui auraient à surveiller tous les messages envoyés et reçus. Ce plan fut transmis au Département d'État américain; ce dernier le reconnut préférable à une prise de possession de l'archipel par la «France libre ${ }^{14}$

La «France libre», non au courant de ces conversations, envoya le capitaine Birot à Saint-Jean, Terre-Neuve; il devait garder un contact étroit avec la situation à Saint-Pierre-etMiquelon. Pendant ce temps, à Londres, le général de Gaulle décida d'agir à la première occasion. Comme l'amiral Muselier aurait à se rendre au Canada pour inspecter le croiseur antisous-marin Surcouf à la base d'Halifax, et comme les corvettes Mimosa, Aconit et Alysse appareillaient pour le Canada en mission d'escorte, le général de Gaulle donna l'ordre à l'amiral Muselier d'effectuer l'opération. ${ }^{15}$ La Mimosa, ayant à bord l'amiral Muselier, appareilla pour Saint-Jean le 2 décembre, et y arriva le 9 décembre. On y établit un dépôt d'approvisionnement et l'on entama des entrevues avec les autorités de Terre-Neuve. Ces dernières approuvèrent l'opération à Saint-Pierre-et-Miquelon; mais l'amiral Muselier crut bon de consulter les autres gouvernements intéressés. Le 9 décembre, il envoya le télégramme suivant au général de Gaulle: «J'appareillerai pour Halifax ... Je suis prêt à faire l'opération de Saint-Pierre-et-Miquelon. Devant nouvelle situation générale, je me rendrai immédiatement à Ottawa pour prendre accord Canada et Amérique. Je vous demande de demander l'accord des Britanniques et de vouloir bien m'en communiquer le résultat. ${ }^{16}$ L'amiral Muselier arriva à Ottawa le 15 décembre et conféra immédiatement avec les hautes autorités canadiennes. Pour obtenir l'approbation des plans de la « France libre », il invoqua les motifs suivants: $1^{\circ}$ le danger pour les convois atlantiques que représentaient le câble et les communications radio reliant

14 Moffat, The Moffat Papers, 358 et passim.

15 De Gaulle, Mémoires de Guerre: 184-185. Muselier, dans De Gaulle, 252 , observe que «le Général me laissa entièrement carte blanche et il précisa qu'il prenait la responsabilité entière des conséquences de l'opération si je décidais de l'entreprendre. 》 Voir supra, note 7.

16 De Gaulle, Mémoires de Guerre: 490. 
Saint-Pierre et Vichy ; $2^{\circ}$ le désir de la population de Saint-Pierreet-Miquelon de s'allier à la "France libre »; $3^{\circ}$ l'oppression que l'administration de l'île, sous l'autorité de Vichy, faisait subir à la population; $4^{\circ}$ la facilité avec laquelle les îles pouvaient être prises; $5^{\circ}$ la contribution que les installations et la maind'œuvre des îles libérées pourraient apporter à la cause alliée; $6^{\circ}$ le vent d'espoir qui secouerait la France; et $7^{\circ}$ la valeur de propagande mondiale d'une telle action. ${ }^{17}$

Le projet rencontra des réactions diverses au Canada; une décision finale ne pouvait être prise, répondit-on, qu'après consultation des autorités américaines. Ottawa et Londres souhaitaient s'informer de la position américaine. Les Anglais avaient reçu une requête du général de Gaulle les priant d'approuver le projet; ils ne voyaient pas d'objection à l'entreprise, avaient-ils répondu, mais exigeaient que tout ordre définitif reçût l'approbation des Etats-Unis. Moffat pria Washington d'exprimer officiellement son attitude pour ensuite en informer l'amiral Muselier et les autorités canadiennes. Après une étude attentive, l'affaire fut transmise au président Roosevelt; le président fut d'avis que l'occupation des îles par la «France libre » constituerait une erreur; il donna néanmoins son approbation à une surveillance des communications de Saint-Pierre avec Vichy, surveillance dont se chargeaient de préférence les Canadiens.

La décision des États-Unis communiquée au général de Gaulle, celui-ci fit savoir officiellement que l'intervention projetée par la «France libre» « ne devait pas être entreprise maintenant $\gg .18$

Au Canada, l'amiral Muselier annonça alors qu'il allait retourner à Saint-Jean et de là s'envoler à Londres. Apparemment telle était bien son intention; un télégramme du général de Gaulle le fit probablement changer d'avis. Le texte de ce télégramme daté du 17 septembre était le suivant: « Nos négociations ici nous ont montré que nous ne pourrons rien entreprendre

17 Muselier, De Gaulle, 259-260.

18 R. E. Sherwood, Roosevelt and Hopkins (New York, 1948), 480. 
à Saint-Pierre-et-Miquelon si nous attendons la permission de tous ceux qui se disent intéressés. Cela était à prévoir. La seule solution est une action de notre propre initiative. Je vous répète que je vous couvre entièrement à ce sujet. ${ }^{19}$

Le jour suivant, le «Foreign Office » avertit officieusement le général de Gaulle que le gouvernement canadien, avec l'approbation des États-Unis ou même à la demande de celui-ci, avait décidé de s'emparer de la station de radio, par force si nécessaire. $^{20}$ Cette information incita le général de Gaulle à l'envoi d'un second télégramme: "Nous savons, de source certaine, que les Canadiens ont l'intention de faire eux-mêmes la destruction $\mathrm{du}$ poste radio de Saint-Pierre. Dans ces conditions, je vous prescris de procéder au ralliement de Saint-Pierre-et-Miquelon par vos propres moyens et sans rien dire aux étrangers. Je prends l'entière responsabilité de cette opération, devenue indispensable pour conserver à la France ses possessions françaises.» ${ }^{21}$ L'amiral Muselier répondit: «Vos ordres seront exécutés aussi rapidement que possible. $\gg^{22}$

\section{ACTION}

Le 24 décembre, à trois heures d'un matin glacial, les corvettes Mimosa, Alysse et Aconit et le croiseur anti-sous-marin Surcouf s'engagèrent dans le chenal séparant les îles SaintPierre-et-Miquelon. Les 25 hommes désignés pour le débarque-

19 De Gaulle, Mémoires de guerre: $492-494$.

20 Ibid: 185.

21 Ibid: 494. Les raisons données par de Gaulle justifiant l'opération à Saint-Pierre-et-Miquelon ont été présentées plus haut. Plusieurs commentateurs voient des motifs divers dans la décision de de Gaulle. De $\mathrm{Ke}-$ rillis, De Gaulle dictateur (Montréal, 1945), 127, note que le "Général de Gaulle, pour des raisons de prestige personnel et pour essayer d'effacer le souvenir persistant de son échec lamentable à Dakar... jugeait utile de faire le coup le plus vite possible. \-Thompson, Two Frenchmen: Pierre Laval and Charles de Gaulle (London, 1951), 184-185, opine que le projet de passer à l'attaque à Saint-Pierre-et-Miquelon fut saisi parce qu'il y avait de bonnes chances de brouiller Muselier avec les Américains et les Anglais. - Herbert Lǔthy, "France in Person», (Encounter, VIII (May, 1957), 58-67) : 66, est d'avis que «l'action fut également décidée pour démontrer qu'en toute matière concernant les affaires françaises, l'Amérique avait à traiter exclusivement avec de Gaulle ».

22 Muselier, De Gaulle, 265. 
ment furent transférés par canot du Surcouf à la Mimosa. Les bâtiments accrurent ensuite leur vitesse afin de mouiller à SaintPierre avant l'aube. Ils y abordèrent par le nord plutôt que par la route normale de l'est afin d'échapper aux postes d'observation.

A l'entrée du port, la Mimosa arrêta un bateau postal en direction de Miquelon; le pilote offrit volontiers ses services. On laissa passer un petit chalutier. L'entrée dans le port s'accomplit immédiatement et la Mimosa reçut l'ordre de s'assurer la surveillance des douanes; une deuxième corvette, l'Alysse, s'empara du vieux bâtiment des douanes, l'Aconit se dirigea rapidement vers l'embarcadère du port d'où elle pouvait couvrir la Mimosa. Quant au Surcouf, on lui réserva la garde de l'entrée du port.

Les armes étaient prêtes à faire feu, bien qu'en première ligne intentionnellement chargées à blanc. Les commandants des navires et les chefs de sections avaient reçu l'ordre de n'utiliser les munitions qu'en cas de nécessité absolue et de légitime défense. La rapidité de l'opération, avait-on prévu, ne donnerait guère aux autorités de l'île le temps d'organiser une résistance, à moins qu'une compagnie n'eût été envoyée de la Martinique. (Mais il eût été difficile pour Vichy d'envoyer des troupes à l'insu des services d'espionnage de la «France libre ».) Ainsi donc, la chance était grande de ne rencontrer que des partisans de la «France libre ${ }^{23}$

Un gendarme qui vit la corvette Mimosa amarrée au bassin, se soumit immédiatement; un officiel qui apparut quelques instants après, fit de même. Grâce au froid perçant et à l'heure matinale, aucune difficulté ne s'offrit; en moins de trente minutes la sécurité de l'île était assurée. On saisit rapidement la centrale téléphonique, puis la station de radio, puis le poste du câble transatlantique et la maison des douanes et le commissariat de police et les bureaux du gouvernement. On se rendit maître

${ }^{23}$ Les deux sources suivantes fournirent principalement la documentation sur ce point, Muselier, De Gaulle, 267-268 et passim, et le rapport d'Ira Wolfert, dans le New York Times (25 décembre 1941 - 11 janvier 1942). 
des remorqueurs Bearn et Caldonia, puis on s'empara de deux canons, de quatre canons à mitraille avec munitions, d'environ 130 autres armes, ainsi que de la goélette Maryse- $R$ et de deux vedettes. Le correspondant du New York Times, Ira Wolfert, rappelle: "Tout ne fut que roses et victoire... sans qu'un seul homme ne fut tué.» ${ }^{24}$

Dans leur hâte de répondre au signal «Exécutez la mission ordonnée », transmis en morse depuis les bâtiments, les hommes qui venaient de débarquer ne firent tout d'abord pas attention aux habitants enthousiastes qui s'étaient levés dans la nuit froide et qui se pressaient à présent sur la route longeant les bassins, criant «Vive de Gaulle » et «Vive l'amiral Muselier ». L'amiral Muselier leur adressa la déclaration suivante au nom de tous ses hommes: «Habitants de Saint-Pierre-et-Miquelon: conformément aux ordres du général de Gaulle, je suis venu pour vous permettre de participer librement et dans l'ordre au plébiscite que vous réclamez depuis si longtemps. Vous aurez à choisir entre la cause de la France libre et la collaboration avec les puissances qui affament, humilient et martyrisent notre patrie. Je ne doute pas que le plus ancien de nos territoires d'outremer, se rangeant aux côtés de la Grande-Bretagne, des Etats-Unis, du Canada, des autres Alliés, ne manifeste en masse sa fidélité aux traditions d'honneur et de liberté qui ont toujours été l'orgueil de la France. Vive la France! Vivent les Alliés ! ${ }^{25}$

Un plébiscite s'organisa pour le lendemain, fête de Noël; tous les hommes de 18 ans et plus y purent participer. ${ }^{26}$ On procéda ensuite à l'arrestation de tous ceux qu'on soupçonnait agents

${ }^{24}$ Close-Up.

25 Cité dans R. Aghion, L'Epopée de la France combattante, (New York, 1943), 166-167.

${ }^{26}$ Lamiral Muselier annonça le résultat du plébiscite à $17 \mathrm{~h} .30$ le jour de Noël; il y assurait $98 \%$ des votes pour la « France libre ». Cité dans Aghion, l'Epopée, 167, et ailleurs. Thomson, Two Frenchmen, 186 met en doute la validité de ce plébiscite. Il remarque que plus d'un tiers des électeurs s'abstint devant le choix \& France libre » ou \& Puissance de l'Axe \$; certains supprimèrent le mot \&libre $>$ et votèrent simplement pour la « France»; d'autres écrivirent 《Vivent Pétain et de Gaulle * (ces bulletins furent annulés), tandis que onze votèrent pour les forces de l'Axe. De Bournat croit que beaucoup s'abstinrent parce que le résultat ne leur paraissait pas douteux. Close-Up. 
de Vichy. Les onze gendarmes locaux, les seuls habitants dont on aurait pu attendre quelque résistance, rendirent les armes sans protester et s'offrirent aussitôt à participer à l'arrestation d'autres suspects, principalement parmi les autorités officielles de l'île. On se hâta d'accepter l'offre de ces gendarmes.

Parmi les officiels mis en état d'arrestation, se trouvait le gouverneur de l'île, le baron de Bournat. Alors qu'on le conduisait pour détention sur le Surcouf, la population lui jeta au visage, «Vive de Gaulle ». Il aurait répondu, «Vive Pétain !».

L'amiral Muselier rassembla les autorités locales et demanda à chacun de rester à son poste et d'assurer le bon fonctionnement de son service; l'enseigne Alain Savary de la «France libre » se vit chargé de l'administration temporaire de l'île, en attendant le résultat du plébiscite. ${ }^{27}$ Ensuite, plusieurs prisonniers, soupçonnés de fidélité à Vichy, furent amenés. "Comme cadeau de Noël, leur dit l'amiral Muselier, la France libre vous offre ce qu'elle peut vous accorder: la liberté. » On ne priva de sa liberté qu'Henri Moraze, boutiquier local et agent reconnu de Vichy. L'envahisseur hissa le symbole de la «France libre », la croix de Lorraine, à la hampe de la maison du Gouverneur aux côtés du drapeau tricolore, tandis que la Marseillaise et la Marche Lorraine étaient jouées à bord de l'Alysse. La population enthousiaste accueillit ce geste par de vibrantes acclamations.

\section{REACTION}

La réaction à la prise de l'archipel Saint-Pierre-et-Miquelon par les forces de la «France libre» ne fut pas lente à se manifester à travers le monde. La déclaration faite par le secrétaire d'Etat américain, Cordell Hull, avec l'approbation du président Roosevelt, est des plus significative : « Nos premiers rapports indiquent que l'intervention effectuée à Saint-Pierre-etMiquelon par trois bâtiments appartenant soi-disant aux forces

27 Il fut nommé plus tard représentant permanent de la $\ll$ France libre » au conseil consultatif de l'île. Voir de Gaulle, Mémoires de guerre: 520, et C. C. Lingard et R. G. Trotter, Canada in World Affairs: septembre 1941 - mai 1944 (Toronto, 1950), 128. 
de la France libre constitue une action contraire à l'accord de toutes les parties intéressées et certainement sans que le gouvernement des États-Unis en ait eu connaissance ou ait exprimé son approbation. Ce gouvernement s'est enquis auprès du gouvernement canadien aux fins de connaître les mesures que ce dernier envisage en vue de restaurer le statu quo dans l'archipel. $\gg^{28}$

Hull se montrait en faveur d'une intervention canadienne pour la restauration du statu quo; on en informa Mackenzie King. Ce dernier entama aussitôt des communications téléphoniques avec le Royaume-Uni et les États-Unis en vue d'une attitude commune. Dans son journal il écrivait, « Nous procéderions à toute action qui recevrait le soutien de Churchill et Roosevelt... ce qui fut accompli à Saint-Pierre par les Français de la France libre, le fut non seulement sans instruction de notre part, mais également en opposition directe à notre politique. $\gg^{29}$ King invoquait d'autres motif's. "Nous décidâmes que toute intervention unilatérale de notre part constituerait l'aveu de notre responsabilité dans l'opération française $\gg .^{30}$

28 Déclaration officielle du Département d'Etat faite le 25 décembre 1941, rapportée dans le Council on Foreign Relations, Documents on American Foreign Relations (23 vol., Boston, 1942), IV: 466. - Cordell Hull, The Memoirs of Cordell Hull (New York, 1948), 1130, donne quelques renseignements précieux sur ses réactions privées et officielles à l'intervention de la «France libre ». Document qui mentionne également l'approbation par le Président de la déclaration du Département d'Etat. - Moffat, The Moffat Papers, 364-365 et passim, rapporte que Hull lui téléphona à Ottawa et lui demanda de persuader les Canadiens de prendre les mesures nécessaires à la restauration du statu quo l'après-midi même. Moffat s'exécuta aussitôt et la réponse des autorités canadiennes lui parvint plus tard dans la journée: «Le Canada ne peut être tenu responsable de l'occupation de Saint-Pierre-et-Miquelon par des forces de la France libre. Nous avons entretenu des contacts étroits avec les Etats-Unis et le Royaume-Uni sur ce point et avons toujours été prêts à coopérer à l'élaboration d'une politique commune. Nous refusons de nous engager à intervenir ou de passer à l'action tant que pareille politique ne se sera pas mise d'accord. Dans ces circonstances et à moins que l'occasion ne se présente de considérer une attitude commune avec le Président et Monsieur Churchill, le Gouvernement canadien ne peut prendre les mesures requises pour l'expulsion des forces de la France libre et la restauration du statu quo dans l'archipel.»Voir aussi Sumner Welles, Seven Decisions that Shaped History (New York, 1950), 62-63, et Pickersgill, The Mackenzie King Record, I: 320.

29 Ibid., I: 320.

30 Ibid. 
Le gouvernement de Vichy lança le communiqué suivant: "les milieux officiels français ont reçu avec satisfaction la nouvelle de la condamnation politique par le gouvernement fédéral de Washington de l'initiative prise par l'ancien amiral Muselier, sans qu'il n'en ait eu connaissance ou exprimé son approbation. $\gg^{31}$ L'incident rendit plus difficile la position de l'ambassadeur des États-Unis à Vichy, William Leahy. La mission diplomatique de ce dernier consistait à s'assurer que le gouvernement de Vichy observait les termes de l'accord intervenu lors de l'armistice; mais après la prise de possession de l'archipel, le maréchal Pétain et l'amiral Darlan étaient en droit de faire observer aux États-Unis qu'ils avaient rompu l'accord prévoyant le maintien du statu quo, c'est-à-dire de la souveraineté française dans les colonies de l'hémisphère occidental. Darlan informa Leahy au début de janvier que les Allemands s'étaient proposé d'envoyer des troupes en Afrique du Nord afin d'empêcher tout renouvellement de pareil incident dans cette région, décision que les Américains visaient précisément à éviter par le maintien de liens étroits avec Vichy. ${ }^{32}$

Le jour de Noël 1941, Mackenzie King alla conférer à Washington avec Roosevelt, Hull et Churchill (ce dernier alors aux États-Unis). L'incident Saint-Pierre-et-Miquelon se trouvait à l'ordre du jour. "Le Président et Churchill, écrivait King, passèrent en revue la situation en Afrique et exprimèrent la nécessité de clore cet incident avant qu'il ne prenne de sérieuses proportions.»33 La première proposition de Roosevelt, soit la formation d'une commission composée d'un représentant de Vichy, de la «France libre» et du Canada, et comportant la restauration du Gouverneur déposé, fut bientôt abandonnée, et ce, en raison des difficultés incontestables que constituait le maintien de Vichy dans les îles et aussi du coup que cela porterait à de Gaulle.

Le 27 décembre, l'ambassadeur de Vichy, Henry Haye, envoya le communiqué suivant à la presse: «Il n'y a pas de

31 Sherwood, Roosevelt and Hopkins, 482.

32 W. D. Leahy, I Was There (New York, 1950), 75.

33 Pickersgill, The Mackenzie King Record, I: 321. 
raison de douter de la restauration du statu quo. Toutes les conversations se poursuivent sur cette base, et je crois qu'une solution satisfaisante pour toutes les parties intéressées peut être atteinte. En ce qui concerne la station radio, elle ne fut jamais employée qu'au service des pêcheurs, mais je crois que les choses pourront être arrangées pour lever tout soupçon quant à la transmission de renseignements dangereux par la radio. $\gg^{34}$

Bien que surpris de ce qui s'était passé aux Iles, les Britanniques étaient vivement opposés à l'expulsion de la «France libre » des îles, spécialement à cause du mécontentement public que pareille action entraînerait à coup sûr. S'adressant à la Chambre des Communes canadienne le 30 décembre, en présence du ministre de Vichy et du représentant de de Gaulle, Churchill manifesta un certain mépris pour les «hommes de Vichy» et loua la «France libre» «tenue dans une estime croissante par neuf personnes sur dix de l'heureux et souriant pays de France d'autrefois $\gg .{ }^{35}$

Soutien et condamnation de l'attitude du Département d'État, voilà ce qui caractérisait et la presse américaine et la presse étrangère. Freda Kerchwey, dans un éditorial de la « Nation 》, accusa le Département d'État de «poursuivre avec un entêtement ridicule sa politique de complaisance vis-à-vis de Vichy ... et... d'affront vis-à-vis de la France libre ». Dans «la répudiation de la France libre à Saint-Pierre-et-Miquelon... elle voyait le symbole le plus effrayant et le plus récent ... de ... notre déchéance morale $\otimes^{36}$

Cordell Hull expliqua son attitude, telle qu'émise dans son communiqué, par l'embarras où se trouvaient engagés les ÉtatsUnis dans trois domaines: $1^{\circ}$ par les relations avec Vichy ( qui nous permirent d'user de notre influence pour empêcher la

34 Goodrich, Documents, IV : 466.

35 Canada, House of Common Debates, le 30 décembre 1941, cité dans Lingard et Reginald, Canada in World Affairs, 126. Il est intéressant de noter que Hull, Memoirs, 1130, considérait comme un \& fait $\gg$ le « soutien non avoué du gouvernement britannique à l'opération > malgré les nombreux documents prouvant que de Gaulle ordonna la prise de possession sans l'accord d'aucun gouvernement.

${ }^{36} \mathrm{~F}$. Kerchwey, «onsieur Hull devrait résigner », (Nation, CLIV, le 3 janvier 1942, 1-2). 
flotte et les bases françaises de tomber aux mains des Allemands et maintenir des observateurs en France et en Afrique du Nord et occidentale française $\gg), 37$ relations qui, onze jours auparavant seulement, avaient été renforcées par l'entente prévoyant « le maintien du statu quo des possessions françaises dans l'hémisphère occidental $\gg^{38} ; 2^{\circ}$ par l'entente avec l'amiral Robert de Vichy visant au même but, et $3^{\circ}$ par l'entente conclue à La Havane entre les Républiques américaines où l'on s'oppose au transfert de souveraineté, de possession, au sujet de tout territoire détenu par les puissances européennes dans l'hémisphère occidental. ${ }^{39}$

Un éditorial du Commonweal donna son approbation totale aux propos de Cordell Hull; les relations avec Vichy, soutenait-on, étaient nécessaires «afin d'assurer le contact avec les peuples conquis et ainsi encourager et raffermir leur opposition à l'envahisseur ». Le judicieux accord entre cette attitude sur le plan diplomatique et l'aide matérielle accordée au général de Gaulle était, aux yeux du Commonweal, mis en danger par la prise de possession de Saint-Pierre-et-Miquelon. ${ }^{40}$

L'amiral Robert de Vichy déclara qu'à son avis les ÉtatsUnis étaient « tenus d'obtenir le rétablissement de la souveraineté de la France de Vichy sur Saint-Pierre-et-Miquelon $\gg .{ }^{41}$ Cordell Hull favorisa vivement cette opinion; il proposait une surveillance canadienne des moyens de communications des îles et une retraite de la part de la «France libre », qui sauverait les apparences. ${ }^{42}$ Le 10 janvier, le New York Times, trop hâtivement, rapportait «qu'une proposition avait été soumise au Comité de la France libre à Londres par le Département d'État requérant le retrait de la France libre ... et... l'évacuation rapide... de ses troupes... de l'archipel Saint-Pierre-et-Miquelon ».

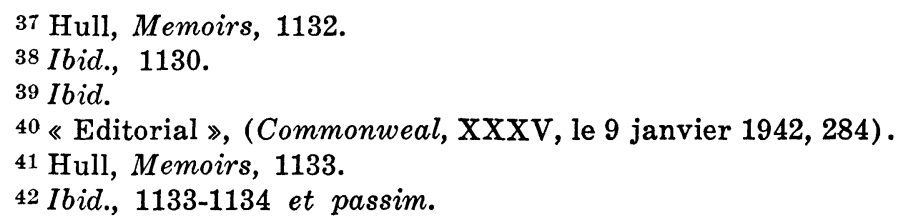


Mais ni de Gaulle ni Churchill ne devaient accepter cette proposition. Roosevelt, pour sa part, n'osa pas insister; on aboutit à une impasse. ${ }^{43}$ Entre-temps, l'amiral Muselier proclamait qu' « aucune puissance au monde ne pourrait nous chasser vivants, mes hommes et moi, de l'île. Pour l'honneur je résisterai à la flotte de n'importe quelle puissance ou combinaison de puissances $\gg{ }^{44}$ Et six jours plus tard, l'amiral Muselier réaffirmait: «Ici, nous sommes, ici, nous resterons. ${ }^{45}$

Les pourparlers continuèrent entre Washington et Londres à un échelon élevé. L'impasse finit par irriter Roosevelt, au point, finit-il par faire savoir à Churchill, qu'il serait capable d'envoyer le cuirassé Arkansas pour déloger de l'île les hommes de la «France libre» ou encore ordonner un blocus jusqu'à ce qu'ils se soumettent. ${ }^{46}$ Finalement, le 22 janvier, Churchill rencontra de Gaulle et lui soumit un projet de solution qu' " avec la plus grande force » il pria le Général d'accepter. ${ }^{47}$ Le projet prévoyait entre autres points importants: $1^{\circ}$ la constitution d'un conseil consultatif, par le truchement duquel serait rétabli un semblant de souveraineté de Vichy; $2^{\circ}$ une surveillance des autorités américaines et canadiennes sur le système de communications de l'archipel; $3^{\circ}$ une autorisation à la force navale de la «France libre» de reprendre à Saint-Pierre ses missions normales, et enfin l'offre des gouvernements américains et canadiens de leur aide économique et autre aux îles. ${ }^{48}$ Churchill ajouta officieusement, après la publication de ce document, qu'il

43 Hull, Memoirs, 1133-1137 et passim. On trouve un aperçu de la fermeté de de Gaulle dans un incident qu'il rappelle en ses Mémoires de guerre: 186-187. "Comme je refusais une pareille solution, M. Eden m'annonça que les Etats-Unis songeaient à envoyer à Saint-Pierre un croiseur et deux destroyers. 'Que ferez-vous en ce cas'? me dit-il. 'Les navires alliés', répondis-je, 's'arrêteront à la limite des eaux territoriales françaises et l'amiral américain ira déjeuner chez Muselier qui en sera certainement enchanté'. - 'Mais si le croiseur dépasse la limite ?' - 'Nos gens feront les sommations d'usage'. 'S'il passe outre ?' 'Ce serait un grand malheur, car, alors, les nôtres devraient tirer'. 》

44 The New York Times, le 5 janvier 1942.

45 Ibid., le 11 janvier 1942.

46 Sherwood, Roosevelt and Hopkins, 488-489.

47 De Gaulle, Mémoires de guerre, 187.

48 Ibid., 519, et Sherwood, Roosevelt and Hopkins, 489. 
aurait pour effet d'atténuer les intérêts extérieurs en conflit à l'île et de renforcer considérablement l'autorité du représentant de la «France libre» au Conseil consultatif. Le général de Gaulle et le Comité national français acceptèrent le projet; mais le statut de l'archipel ne connut de changement qu'après la libération de la France et la formation d'un nouveau gouvernement français.

Ainsi, « un fait qui menaça d'être un chapitre dans l'histoire, finit par n'être qu'un incident $\gg^{49}$

WILLIAM HANNA, Assistant-Professor, Michigan State University, East Lansing, U.S.A.

49 Hull, Mémoirs, 1137.

\section{HISTORIAN CLAIMS CABOT LANDED IN NEWFOUNDLAND}

PROVIDENCE, R.I. (AP) - A Naval historian from the Smithsonian Institute Saturday presented a paper at a meeting of the Society for the History of Discoveries at Brown University which indicated that John Cabot, English explorer, landed on Newfoundland in 1497 and not on Cape Cod or in the Baffin Bay area as some historians contend.

The paper, entitled "The Labrador Landfall of John Cabot: the 1497 Voyage, Reconsidered," was presented by Melvin H. Jackson of the Institute's Division of Naval History. Jackson analyzed available navigational data in arriving at his conclusion.

About 50 persons attended the society's meeting in the John Carter Brown Library. The society was founded two years ago at a meeting of the American Historical Association. Its purpose is to study the history of various discoveries throughout the world.

Extrait de Portland, Maine, Sunday Telegram, Oct. 28, 1962. 